ARTÍCULO ORIGINAL

\title{
IMPACTO ECONÓMICO FINANCIERO DEL CUMPLIMIENTO DE NORMAS TRIBUTARIAS EN LA GESTIÓN EMPRESARIAL
}

\author{
FINANCIAL ECONOMIC IMPACT OF TAX STANDARDS COMPLIANCE \\ IN CORPORATE MANAGEMENT
}

\author{
Karina Esther Alva Suárez \\ Magister en Política y Gestión Tributaria de la Universidad Nacional Mayor de San Marcos. \\ Correo: kalvas@tramarsa.com.pe (autor corresponsal).
}

[Recibido: 2016/11/15 Aceptado: 2017/01/14]

\section{RESUMEN}

En relación a la Ley del Impuesto a la Renta, menciona que son deducibles los gastos que son causales para la generación de la renta gravada, la misma que deben cumplir determinados requisitos formales y sustanciales. Sin embargo existe la problemática de que las empresas pierden el derecho de la deducción de sus gastos y otros beneficios fiscales, por no contar con políticas y procedimientos de control, así como capacitación, lo que impacta en su gestión económica y financiera. El presente trabajo se desarrolla mediante estudios descriptivos (observación de los hechos) y estudios correlacionales (medición del grado de relación que existe entre las variables). El método empleado es inductivo, puesto que del estudio de un caso particular. Determinándose que el incumplimiento de las normas tributarias por una falta de procedimientos y políticas de control; así como la falta de capacitación de los miembros de la organización impacta en forma negativa en los resultados económicos (rentabilidad) y financieros (liquidez) de la organización.

\section{PALABRAS CLAVE}

Gastos deducible, gestión económica; gestión financiera; políticas, procedimientos, capacitación.

\begin{abstract}
In relation to the Income Tax Law, it mentions that the expenses that are causal for the generation of taxable income, which must meet certain formal and substantial requirements, are deductible. However, there is the problem that companies lose the right to deduct their expenses and other tax benefits, because they do not have control policies and procedures, as well as training, which impacts on their economic and financial management. The present work is developed through descriptive studies (observation of the facts) and correlational studies (measurement of the degree of relationship that exists between the variables). The method used is inductive, since it is the study of a case. Determining that non-compliance with tax rules due to a lack of control procedures and policies; as well as the lack of training of the members of the organization negatively impacts the economic (profitability) and financial (liquidity) results of the organization.
\end{abstract}

\section{KEYWORDS}

Deductible expenses, economic management; financial management; policies, procedures, training.

Como Citar: Alva, K. (2017). Impacto económico financiero del cumplimiento de normas tributarias en la gestión empresarial. Quipukamayoc, 25(48), 19-25. doi: http://dx.doi.org/10.15381/quipu.v25i48.13987

(c) Los autores. Este artículo es publicado por la Revista Quipukamayoc, Universidad Nacional Mayor de San Marcos. Este es un artículo de acceso abierto, distribuido bajo los términos de la Licencia Creative Commons Atribución-NoComercial-Compartirlgual 4.0 Internacional.(http://creativecommons.org/licenses/by-nc-sa/4.0/), que permite el uso no comercial, distribución y reproducción en cualquier medio, siempre que la obra original sea debidamente citadas. 


\section{INTRODUCCIÓN}

El artículo surge a raíz de la experiencia laboral en campo tributario en el sector empresarial y de ver como las compañías se ven afectadas económica y financieramente por los costos fiscales innecesarios, los cuales inciden directamente en sus resultados.

Por ello el presente trabajo cobra importancia ya que necesario que las empresas implementen procedimientos y políticas de control tributario así capacitación vinculada al cumplimiento de las normas a fin de evitar los sobrecostos tributarios. Así mismo llevar un control que les permita monitorear en forma periódica y tomar las correcciones en forma oportuna.

El objetivo de este trabajo es demostrar que el incumplimiento de las normas tributarias, tienen un impacto negativo en la gestión de la empresa, tanto económicos (rentabilidad) como financieros (liquidez).

El presente trabajo se justifica ya que las empresas deben tributar en forma justa y correcta, es decir el impuesto debiera gravar sus utilidades y no sobre costos adicionales que pudieran ser ahorrados.

Se ha revisado en forma preliminar trabajos de investigación relacionado a la impacto económico y financiero del cumplimiento de normas tributarias por lo que es importante mencionar y comentar algunos trabajos de investigación que fueron un aporte importante en el desarrollo del presente estudio.

Salinas (2000) señala que el régimen de infracciones, sanciones y delitos reviste alta complejidad y sus permanentes cambios, no contribuye al buen entendimiento y cumplimien- to de sus obligaciones, por tanto esta debería simplificarse y difundirse mediante un adecuando plan de capacitación.

Valencia (2012) menciona que la implementación de un programa educativo de tributación es necesaria para formar conciencia tributaria en los contribuyentes. Asimismo propone que el Estado de incluir como cursos obligatorios en las escuelas curso de cultura tributaria puesto que finalmente todos serán en algún momento contribuyentes

Cayo (2000) sostiene que los impuestos son incompatibles con la capacidad contributiva de las personas naturales, ya que no permite efectuar deducciones inherentes a la formación de sus rentas.

Falcón (2002) concluye que las sanciones tributarias en el Perú así como la de otros países latinoamericanos son de niveles muy altos en comparación a la infracción incurrida por el contribuyente, siendo el recargo de intereses una sanción sobre sanción. Otro punto importante es su propuesta de que las multas deberían ser gastos deducibles.

Angulo (2011) manifiesta un análisis de tratamiento contable de la NIC 12 Impuesto a la Renta recomendando que el impuesto a renta del ejercicio debe mostrarse en resultados en forma separada el impuesto corriente y el diferido, y no neteada ya que esto permitiría una mejor lectura y control del mismo. Consideramos que lo señalado por el autor es muy importante puesto que ambos cálculo tiene impactos y tratamientos diferentes.

Recavarren (2007) menciona que la Contabilidad de Gestión es una herramienta que ayuda en las empresas a agregar valor, tener una mejor planificación así como tomar decisiones estratégicas para la organización.

Las Normas Tributarias en el Perú (El Impuesto a la Renta)

En relación al Impuesto a la Renta, es el tributo recaudado más importante en el Perú puesto que ello financia el gasto público.

Mullin (1978) explica que el impuesto a la renta que grava la totalidad de las rentas obtenida cualquiera sea su procedencia o destino con tasas progresivas que hagan más fuerte la carga relativa a medida que mayor sea la renta total, atendiendo a características como la globalidad, personalidad y progresividad.

Alva (2012), hace una cita textual de Bravo Cucci quien menciona que El Impuesto a la Renta es un tributo que se precipita directamente sobre la renta como manifestación de riqueza. En estricto, dicho impuesto grava el hecho de percibir o generar renta, la cual puede generarse de fuentes pasivas (capital), de fuentes activas (trabajo dependiente o independiente) $\mathrm{o}$ de fuentes mixtas (realización de una actividad empresarial $=$ capital + trabajo).

Actualmente está normado por el Decreto Supremo No 179-2004 EF y por su Reglamento 122-94-EF. Es un impuesto directo que grava los ingresos después de efectuar las deducciones de gastos permitidos llamados también gastos deducibles.

En relación a estos gastos permitidos, Bustamante (2008) desarrolla lo indicando por el Artículo $\mathrm{N}^{\circ} 37$ de la Ley del Impuesto a la Renta y nos menciona que para proceda la deducción 
de un gasto este debe cumplir con el principio de causalidad, lo cual incluye los siguientes criterios: deben ser razonables, necesarios, proporcionales y además generales. Adicionalmente a ello que cumplir con el principio de devengado y fehaciencia, ello en concordancia con el Artículo $57^{\circ}$ de la Ley.

En resumen, el contribuyente debe prestar atención, ya que no basta con efectuar los desembolsos que le son necesarios para la generación y mantenimiento de su fuente generadora de renta, sino que debe observar y cumplir esta serie de condiciones a fin de que pueda deducir de los ingresos gravables y tributar sobre una base. No se trata de que sea justo o injusto el desconocimiento de un gasto, sino que en cada transacción que la empresa genera, todo el personal involucrado en ello, debe tener conocimiento de estos requisitos (tanto formales como sustanciales) vale decir que debe estar sensibilizados mediante capacitación y procedimientos de control establecidos.

\section{La Gestión Empresarial y su relación con los tributos}

Todas las empresas tienen un modelo de gestión empresarial, lo cual le permitirá en el tiempo alcanzar sus objetivos de productividad, sostenibilidad y competitividad.

La gestión empresarial es de suma importancia ya que está relacionada a las funciones de planificación, organización, dirección y control.

Es importante distinguir en que consiste la gestión empresarial, desde el punto de vista económico y financiero, y además cual es la relación de ambos con los tributos.
La Gestión Económica, está relacionada a los resultados de la empresa, es decir como gestiona sus ingresos, gastos y costos, la ganancia y las pérdidas.

El accionista o inversor es quien al final de cada ejercicio revisará los resultados del negocio y obtendrá sus dividendos. Es decir a mayor ganancia, mayor distribución de dividendos.

Apaza (2011) manifiesta que la rentabilidad de una empresa se mide luego de haber realizados sus actividades $y$ haber remunerado los demás factores, siendo así es capaz de generar ganancias a ser repartido entre sus accionistas.

Ahora bien, antes de efectuar esta distribución a los accionistas, se deberá deducir el impuesto correspondiente, es decir mayor impuesto a la renta, menor importe a distribuir para el inversionista.

La Gestión Financiera, está relacionado a los recursos monetarios, es decir la liquidez. Quién lidera este rol es el área financiera quienes deben velar por la optimización de los recursos que le permitirá tener la solvencia para cubrir los pagos a sus proveedores, trabajadores, al fisco y a sus accionistas.

La relación entre la gestión empresarial y los tributos es muy importante, ya que uno de los principales acreedores es el fisco, es decir el Estado. Por lo tanto la empresa debe gestionar sus recursos de tal modo que le permita cumplir con sus obligaciones mediante el correcto y oportuno pago de sus tributos.

Las Políticas y Procedimientos en la empresa

Son las guías, directrices y lineamientos que establece la empresa y que per- mite implementar el funcionamiento de la organización. Estas políticas y procedimiento se difunden a través de los manuales

Actualmente las empresas por ejemplo para obtener certificaciones ISO, deben cumplir con la elaboración y difusión de manuales de políticas, procedimientos e instructivos sobre diversas actividades de la organización, sin embargo no es común que las empresas apliquen esta misma metodología en el campo tributario, es decir se podría aprovechar estas herramientas para elaborar manuales que contengas procedimientos vinculados a normas tributarias que ayude y oriente el trabajo de los empleados en la correcta sustentación de los gastos así por ejemplo emitir manuales como:

- Manual de procedimientos de Guías de Remisión

- Manual de procedimientos para la Emisión de comprobantes de Pago

- Instructivo para Planillas de Movilidad

La capacitación del personal en normas tributarias

Las normas tributarias son publicadas a través del Diario Oficial el Peruano y si bien el área legal o tributaria de la compañía son los responsables de su lectura, difusión y aplicación, es necesario que las mismas sea explicadas en un lenguaje sencillo y accesible a los demás miembros de la compañía. Considerando que la capacitación es la herramienta para el logro de objetivos de la organización, es indispensable mantener capacitados a los miembros 


\section{MATERIAL Y MÉTODOS}

El presente trabajo se desarrolla mediante estudios descriptivos (observación de los hechos) y estudios correlacionales (medición del grado de relación que existe entre las variables). El método empleado es inductivo, puesto que del estudio de un caso par- ticular, los resultados se tomarán para conclusiones de carácter general.

Teniendo como unidad de análisis al área de contabilidad y finanzas. Con una población, objeto de estudios correspondiente a las empresas operadoras portuarias del sector privado. Considerando como muestra a una

Tabla 1.

Detalle del cálculo del Impuesto a la Renta Corriente

Fuente: Elaboración propia en base a las Declaraciones Juradas de renta de los años 2014, 2015 y 2016

\begin{tabular}{|c|c|c|c|c|}
\hline \multicolumn{5}{|l|}{ (Expresado en soles) } \\
\hline Periodos & 2014 & 2015 & 2016 & Total General \\
\hline Utilidad Comercial & 28829944 & 20890871 & 98505896 & 148226710 \\
\hline \multicolumn{5}{|l|}{ Adiciones Permanentes } \\
\hline $\begin{array}{l}\text { a. Boletas de venta no pertenecen } \\
\text { al Nuevo }\end{array}$ & 124568 & 116433 & 110571 & 351572 \\
\hline $\begin{array}{l}\text { b. Comprobantes que no cum- } \\
\text { plen con los requisitos de la } \\
\text { Ley de Comprobantes de Pago }\end{array}$ & 344996 & 283276 & 51418 & 679690 \\
\hline $\begin{array}{l}\text { c. Donaciones no Deducibles y } \\
\text { actos de }\end{array}$ & 10177 & 13284 & & 23462 \\
\hline $\begin{array}{l}\text { d. Gastos de Ejercicios } \\
\text { Anteriores no }\end{array}$ & 1656336 & 3559207 & 290464 & 5506008 \\
\hline e. Gastos de movilidad & 36702 & 48027 & 4241 & 88970 \\
\hline f. $\quad$ Gastos de viaje en país & 1556 & 82099 & & 83655 \\
\hline g. Gastos de Viaje Exterior & 13139 & 12996 & 1464 & 27599 \\
\hline $\begin{array}{l}\text { h. Impuesto a la Renta } \\
\text { Asumido - No }\end{array}$ & 73792 & 167357 & 32946 & 274095 \\
\hline $\begin{array}{l}\text { i. Pérdida Crédito Fiscal - IGV } \\
\text { proveniente de gastos no } \\
\text { deducibles }\end{array}$ & 4713 & 61471 & 32484 & 98668 \\
\hline $\begin{array}{ll}\text { j. } & \text { Sanciones Administrativas } \\
& \text { Fiscales }\end{array}$ & 718332 & 361535 & 81544 & 1161411 \\
\hline k. Baja de activos fijos & 5338 & 50927 & 88807 & 145072 \\
\hline Total adiciones permanentes & 2989650 & 4756614 & 693940 & 8440204 \\
\hline Utilidad tributaria & 31819594 & 25647484 & 99199836 & 8440204 \\
\hline tasa & $30 \%$ & $28 \%$ & $28 \%$ & \\
\hline $\begin{array}{l}\text { Impuesto a la Renta } \\
\text { Corriente }\end{array}$ & 9545878 & 7181296 & 27775954 & 44503128 \\
\hline $\begin{array}{l}\text { Tasa Efectiva (Imp. Renta / } \\
\text { Utilidad Comercial) }\end{array}$ & $33,11 \%$ & $34,38 \%$ & $28,20 \%$ & $30,02 \%$ \\
\hline
\end{tabular}

empresa operadora portuaria, líder en el sector del comercio exterior, bajo un periodo de estudio de 3 últimos años $(2014,2015$ y 2016), que por el volumen de operaciones es factible de ser estudiada y los resultados obtenidos le es aplicable todo el universo de empresas de cualquier sector que tribute impuesto a la renta empresarial.

Los datos que han sido materia de estudio se obtuvieron mediante la revisión de normas tributarias, documentos (información contable y tributaria de la empresa), bibliografía e información en Internet.

\section{RESULTADOS}

Determinación de Impacto económico financiero del cumplimiento de normas tributarias

Durante el periodo bajo estudio (2014, 2015 y 2016), se tomó las declaraciones juradas de impuestos a la renta y se revisó los gastos adicionados como permanentes y que incrementaron la base imponible para la determinación del cálculo del impuesto a la renta.

Se retiró de este análisis las adiciones y deducciones temporales puesto que estas tienen un efecto diferido en el tiempo y se controlan mediante el impuesto a la renta diferido (NIC 12). Es decir el análisis corresponde sólo al impuesto a la Renta Corriente.

En el Tabla 1 se resume en forma comparativa el cálculo del impuesto a la renta de los 3 últimos años, donde se detalla los gastos que la compañía ha incurrido en la generación de la renta (es decir que cumplieron el principio de causalidad), pero que sin embargo por no cumplir con los requisitos exigidos por la normas tributarios (requisitos formales y sustanciales) 
debió adicionarlos a la base imponible en forma permanente, incrementando con ello la base imponible sobre la cual calcula el Impuesto a la Renta.

Explicaciones a las adiciones permanentes mostradas en la Tabla 1

a) Corresponde a las adquisiciones a empresas mediante boletas de venta que no están dentro del Régimen del Nuevo RUS.

b) Gastos por los cuales no se solicitaron los Comprobantes de pago correspondientes.

c) No se gestionaron los certificados de Donación a las empresas Perceptoras de Donaciones.

d) No se provisionaron oportunamente los gastos tanto administrativo como operativos siendo conocidos por la compañía.

e) No se contó con Planillas de Movilidad, sino con comprobantes de caja.

f) No se sustentaron con los Comprobantes del Pago o Declaraciones Juradas del viaje al exterior, sino con comprobantes de caja.

g) No se cuenta con los comprobantes de pago que sustentan el viaje sino con comprobantes de caja.

h) No se informó a los proveedores del exterior que debían efectuarse la retención del Impuesto a la Renta en el Perú.

i) Impuesto General a las ventas vinculado a Gastos de Ejercicios Anteriores no deducibles.

j) Multas relacionadas a envió extemporáneo de Información a la Aduana. k) Se efectuó baja de Activo Fijo obsoleto sin contar con informes técnicos.

A continuación se mostrará los resultados en el base a la información de-

tallada en la Tabla 1, es decir demostraremos los resultados del Impacto Económico y Financiero del incumplimiento de las Normas Tributarias en la Tabla 2

Tabla 2.

Resultado del Impacto Económico Financiero del cumplimiento de las Normas Tributarias Fuente: Elaboración propia en base a las Declaraciones Juradas de Impuesto a la Renta y los Estados Financieros

\begin{tabular}{|c|c|c|c|c|}
\hline \multicolumn{5}{|l|}{ (Expresado en soles) } \\
\hline & 2014 & 2015 & 2016 & Total General \\
\hline Utilidad Comercial & 28829944 & 20890871 & 98505896 & 148226710 \\
\hline (mas) Adiciones Permanentes & 2989650 & 4756614 & 693940 & 8440204 \\
\hline Utilidad Tributaria & 31819594 & 25647485 & 99199836 & 156666914 \\
\hline Impuesto a la Renta Corriente & 9545878 & 7181296 & 27775954 & 44503128 \\
\hline \multicolumn{5}{|l|}{ 1.- Impacto Financiero (Liquidez) } \\
\hline $\begin{array}{l}\text { Imp.Renta en Base a la Utilidad } \\
\text { Tributaria }\end{array}$ & 9545878 & 7181296 & 27775954 & 44503128 \\
\hline $\begin{array}{l}\text { Imp.Renta en base a la Utilidad } \\
\text { Comercial }\end{array}$ & 8648983 & 5849444 & 27581651 & 42080078 \\
\hline
\end{tabular}

\begin{tabular}{|c|c|c|c|c|}
\hline $\begin{array}{l}\text { Mayor pago de Impuesto a la } \\
\text { Renta }\end{array}$ & 896895 & 1331852 & 194303 & 2423050 \\
\hline
\end{tabular}

\section{2.- Impacto Económico (Rentabilidad)}

\begin{tabular}{|c|c|c|c|c|}
\hline Utilidad Comercial & 28829944 & 20890871 & 98505896 & 148226710 \\
\hline Imp.Renta sobre Base Financiera & (8 648983$)$ & (5 849444$)$ & $(27581651)$ & (42080 078) \\
\hline $\begin{array}{l}\text { Utilidad distribuir accionistas } \\
\text { (Financiero) }\end{array}$ & 20180961 & 15041427 & 70924245 & 106146632 \\
\hline Utilidad Tributaria & 28829944 & 20890871 & 98505896 & 148226710 \\
\hline Imp.Renta sobre Base Tributaria & (9 545878$)$ & (7 181296$)$ & (27 775954$)$ & (44 503 128) \\
\hline $\begin{array}{l}\text { Utilidad distribuir accionistas } \\
\text { (Tributario) }\end{array}$ & 19284066 & 13709575 & 70729942 & 103723582 \\
\hline
\end{tabular}

\begin{tabular}{|c|c|c|c|c|}
\hline \multicolumn{5}{|l|}{ Resumen } \\
\hline $\begin{array}{l}\text { Utilidad a distribuir accionistas } \\
\text { (Financiero) }\end{array}$ & 20180961 & 15041427 & 70924245 & 106146632 \\
\hline $\begin{array}{l}\text { Utilidad a distribuir accionistas } \\
\text { (Tributario) }\end{array}$ & 19284066 & 13709575 & 70729942 & 103723582 \\
\hline
\end{tabular}

Menor Distribución Dividendos

$896895 \quad 1331852$

194303

2423050 
Veamos a continuación una explicación de los resultados obtenidos en la Tabla 2

Resultados del Impacto Económico. Se determinó que el Impuesto a la Renta Corriente calculado sobre utilidad tributaria es mayor que el obtenido sobre la base de la utilidad contable, lo cual nos indica que hay un impacto negativo, puesto que hay un pago adicional de impuesto a la Renta de S/. 2423050.

Es te mayo pago se traduce en una disminución en la liquidez, que pudo ser aplicado en inversiones y pagos de obligaciones a otros acreedores.

Resultados del Impacto Financiero

Se determinó que al tener una mayor impuesto a la renta corriente, es me- nor el resultado a distribuir a los accionistas. Es decir se distribuyó dividendos en S/. 2423050 que pudieron ser reinvertidos en la misma empresa en nuevos negocios o mediante aportes adicionales de capital.

Resultado de políticas, procedimientos tributarios y capacitación al personal

En relación a las políticas y procedimientos se determinó que la organización cuentas con Normas ISO:

1. Certificaciones de Gestión de Seguridad Industrial y Salud Ocupacional OHSAS 18001:2007)

\section{Certificación ISO 14001:2004}

3. Certificado en BASC (Bussnisess Alliance for Secure Commerce)

\section{Tabla 3.}

Detalle de Inversión de la empresa en Capacitación para el Personal Fuente: Elaboración propia en base al Libro Mayor de la cuenta de Gastos por Capacitación de la empresa de los años 2014,2015 y 2016

\section{(Importe en soles)}

\begin{tabular}{|c|c|c|c|c|c|}
\hline Capacitación & 2014 & 2015 & 2016 & $\begin{array}{c}\text { Total } \\
\text { general }\end{array}$ & $\%$ \\
\hline Seguridad Portuaria & 151826 & 310936 & 330524 & 793286 & $39,21 \%$ \\
\hline Administracion Empresarial & 164820 & 102103 & 216926 & 483849 & $23,91 \%$ \\
\hline Relaciones Laborales & 61775 & 103763 & 61387 & 226925 & $11,22 \%$ \\
\hline Operación de Equipos Portuarios & 86892 & 49471 & 22624 & 158987 & $7,86 \%$ \\
\hline Gestión ISO & 29433 & 26695 & 22593 & 78721 & $3,89 \%$ \\
\hline Negocios Internacionales & 2881 & 19016 & 43122 & 65019 & $3,21 \%$ \\
\hline Derecho y Talleres Aduanero & 15726 & 27934 & 11942 & 55602 & $2,75 \%$ \\
\hline Cursos de Ingles & 9336 & 13716 & 31762 & 54814 & $2,71 \%$ \\
\hline Gerencia de Proyectos & 15119 & 13600 & & 28719 & $1,42 \%$ \\
\hline Office y ERP SAP & 577 & 4050 & 23213 & 27840 & $1,38 \%$ \\
\hline Finanzas & 14546 & 3140 & 8695 & 26381 & $1,30 \%$ \\
\hline $\begin{array}{l}\text { Normas Internacional Financieras - } \\
\text { NIIF }\end{array}$ & & 4000 & 17708 & 21708 & $1,07 \%$ \\
\hline Tributación & 51 & 102 & 1356 & 1508 & $0,07 \%$ \\
\hline Total general & 552983 & 678526 & 791852 & 2023362 & $100 \%$ \\
\hline
\end{tabular}

No se contaba con ninguna política, procedimiento o instructivo vinculados a temas tributarios relacionados a procedimientos de sustentación de gastos.

En relación a capacitación se revisó los gastos que ha efectuado la empresa en los últimos 3 años $(2014,2015$, y 2016) y se muestran en la Tabla 3 y se puede apreciar que solo un $0,07 \%$ se ha incurrido en capacitación en temas vinculados a cumplimiento de normas tributarias

\section{DISCUSIÓN}

La gestión empresarial abarca dos ámbitos: La gestión financiera vinculada a la rentabilidad de la empresa y la gestión económica vinculada a la liquidez. Estos ámbitos están estrechamente relacionados a la gestión de los tributos. Por lo tanto si no se miden los impactos financieros y económicos ante el incumplimiento de las normas tributarias, a fin de evitar costo fiscales innecesarios por no sustentar debidamente los gastos según lo indicado por las normas genera un mayor pago de impuestos y una menor distribución de dividendos. Es decir se concluye que el incumplimiento de las normas tributarias tienen un impacto negativo en la gestión económica y financiera.

Las empresas si bien implementan y difunden en la organización política $\mathrm{y}$ procedimientos vinculados a certificaciones medioambientales tales como ISO, no implementan esta misma herramienta en los temas tributarios.

Las empresas no incurren en suficientes gastos en capacitación relacionados a normas tributarias, es decir no se cuenta con un plan de capacitación 
interna y externa vinculado al cumplimiento de normas tributarias.

\section{REFERENCIAS BIBLIOGRÁFICAS}

Angulo, L. (2011). Impuesto a la Renta Diferido y sus implicancias tributarias en el Perú. (Tesis de Maestría). Universidad Nacional Mayor de San Marcos, Lima.

Alva M. (2012). El Impuesto a la Renta y las Teorías que determinan su afectación. Revista Actualidad Empresarial (249), 1-6.

Apaza, M. (2011). Estados Financieros Formulación, Análisis e Interpretación. Lima: Editorial Instituto Pacífico SAC.

Bustamante, C. (2008). El principio de causalidad para la deducción del gasto. Lima, Perú. Caballero Bustaman- te. Recuperado de http://studylib.es/ doc/4813738/el-principio-de-causalidad-para-la-deducci\%C3\%B3n-delgasto

Cayo, L. (2000). Principio de Capacidad Contributiva y su Incompatibilidad con el Impuesto a la Renta, Predial y el Régimen Único Simplificado. (Tesis de Maestría). Universidad Mayor de San Marcos, Lima.

Falcón, C. (2002). Las Sanciones Tributarias en el Perú y el pago de impuestos insolutos. (Tesis de Maestría). Universidad Nacional Mayor de San Marcos, Lima.

García, R. (1978). Impuesto sobre la Renta: Teoría y Técnica del Impuesto. Buenos Aires: Centro Interamericano de Estudios Tributarios.
Recavarren, F. (2007). Contabilidad de Gestión herramienta estratégica para la competitividad de las Pymes en el Perú. (Tesis de maestría Mención: Contabilidad de Gestión). Universidad Nacional Mayor de San Marcos, Lima.

Salinas, C. (2000). Análisis del Régimen de Infracciones, sanciones y delitos tributarios en el Perú - Propuestas de cambio para su mejor aplicación. (Tesis de Maestría). Universidad Nacional Mayor de San Marcos, Lima.

Valencia, A. (2012). Influencia de la aplicación de un programa de educativo de tributación en la conciencia tributaria de los Estudiantes de la Facultad de la Universidad Nacional Mayor de San Marcos. (Tesis de Doctorado). Universidad Nacional de Educación Enrique Guzmán y Valle. 